\title{
Implementation of logical gates on infinite dimensional quantum oscillators
}

\author{
Nabile Boussaïd \\ Laboratoire de mathématiques \\ Université de Franche-Comté \\ 25030 Besançon, France \\ Nabile.Boussaideuniv-fcomte.fr
}

\author{
Marco Caponigro \\ Institut Élie Cartan de Nancy and \\ INRIA Nancy Grand Est \\ 54506 Vandœuvre, France \\ Marco.Caponigroeinria.fr
}

\author{
Thomas Chambrion \\ Institut Élie Cartan de Nancy and \\ INRIA Nancy Grand Est \\ 54506 Vandœuvre, France \\ Thomas. Chambrion@inria.fr
}

\begin{abstract}
In this paper we study the error in the approximate simultaneous controllability of the bilinear Schrödinger equation. We provide estimates based on a tracking algorithm for general bilinear quantum systems and on the study of the finite dimensional Galerkin approximations for a particular class of quantum systems, weakly-coupled systems. We then present two physical examples: the perturbed quantum harmonic oscillator and the infinite potential well.
\end{abstract}

\section{INTRODUCTION}

\section{A. Logical gates}

Quantum computation relies on the idea to store an information in the state of quantum system. This state is described by the wave function, that is, a point $\psi$ in the Hilbert sphere of $L^{2}(\Omega, \mathbf{C})$, where $\Omega$ is a Riemannian manifold.

When submitted to an excitation by an external field (e.g. a laser), the time evolution of the wave function is governed by the bilinear Schrödinger equation

$$
i \frac{\partial \psi}{\partial t}=-\frac{1}{2} \Delta \psi+V(x) \psi(x, t)+u(t) W(x) \psi(x, t),
$$

where $V, W: \Omega \rightarrow \mathbf{R}$ are real functions describing respectively the physical properties of the uncontrolled system and the external field, and $u: \mathbf{R} \rightarrow \mathbf{R}$ is a real function of the time representing the intensity of the latter.

When the manifold $\Omega$ is compact, the linear operator $i(\Delta / 2-V)$ admits a set of eigenstates $\left(\phi_{n}\right)_{n \in \mathbf{N}}$.A logical gate, or quantum gate, is a unitary transformation in $L^{2}(\Omega, \mathbf{C})$ for which some finite dimensional space of the form $\operatorname{span}\left\{\phi_{1}, \phi_{2}, \ldots, \phi_{n}\right\}$ is stable. To build a given logical gate $\widehat{\Upsilon}$ from the system (1), one has to find a control law $u$ such that the propagator $\Upsilon_{T}^{u}$ at a certain time $T$ of (1) satisfies $\Upsilon_{T}^{u}\left(\phi_{j}\right)=\widehat{\Upsilon} \phi_{j}$ for every $j=1, \ldots, n$.

The main difficulty with this problem is that the space $L^{2}(\Omega, \mathbf{C})$ has infinite dimension. For the sake of simplicity, one often only considers the case where $\Omega$ is a finite union of points (or, equivalently, $L^{2}(\Omega, \mathbf{C})$ is finite dimensional). Nevertheless, most of the usual quantum systems evolves on non trivial manifolds $\Omega$. This papers deals with the effective implementation of some simple logical gates on models of quantum oscillators on 1-dimensional manifolds.

\section{B. Quantum control}

The problem of driving the solutions of (1) to a given target has been intensively studied in the past decades, both in the finite and infinite dimensional case. Many advances have been done in the infinite dimensional case, when there is only one source and one target. The interested reader may refer, for instance and among many other references, to [5], [7] for the theoretical viewpoint and to [8] for numerical aspects. In particular, it was proved in [3] that exact controllability is impossible in general. This does not prevent to study approximate controllability of (1), that is to replace the condition $\Upsilon_{T}^{u}\left(\phi_{j}\right)=\widehat{\Upsilon} \phi_{j}$ by $\left\|\Upsilon_{T}^{u}\left(\phi_{j}\right)-\widehat{\Upsilon} \phi_{j}\right\| \leq$ $\varepsilon$ for every $j=1, \ldots, n$. To the best of our knowledge, there are only very few results of simultaneous controllability in the infinite dimensional case and the only available effective control techniques have been described in [9] and [11].

Recently, we noticed in [10] that a certain class of bilinear systems are precisely approached by their Galerkin approximations. Two important examples of these so-called weakly-coupled systems are the quantum harmonic oscillator and the infinite potential well. The structure of weaklycoupled systems permits precise numerical simulations for the construction of quantum gates.

\section{Content of the paper}

The theoretical background is recalled in Section II Besides a quick survey on simultaneous control techniques for Equation (1), we give precise definitions and approximation results for weakly-coupled systems. In Section III we apply these results to a perturbation of the quantum harmonic oscillator and we provide estimates for the error in the controllability in a suitable finite dimensional approximation. Similarly, in Section IV we study the infinite potential well.

\section{General theORETICAL RESULTS}

\section{A. Framework and notations}

We reformulate the problem (1) in a more abstract framework. In a separable Hilbert space $H$ endowed with norm $\|\cdot\|$ and Hilbert product $\langle\cdot, \cdot\rangle$, we consider the evolution problem

$$
\frac{d \psi}{d t}=(A+u B) \psi
$$

where $(A, B)$ satisfies Assumption 1 .

Assumption 1: $(A, B)$ is a pair of linear operators such that

1) for every $u$ in $\mathbf{R}, A+u B$ is essentially skew-adjoint; 
2) $A$ is skew-adjoint and has purely discrete spectrum $\left(-i \lambda_{k}\right)_{k \in \mathbf{N}}$, associated to the Hilbert basis $\left(\phi_{k}\right)_{k \in \mathbf{N}}$ of eigenvectors of $A$.

From Assumption 111, one deduces that, for every piecewise constant $u, u: t \mapsto=\sum_{j} u_{j} \chi_{\left(t_{j}, t_{j+1}\right)}(t)$, with $0=$ $t_{0} \leq t_{1} \leq \ldots \leq t_{N+1}$ and $u_{0}, \ldots, u_{N}$ in $\mathbf{R}$, the solution $t \mapsto \Upsilon_{t}^{u} \psi_{0}$ of (2) has the form

$$
\begin{aligned}
\Upsilon_{t}^{u} \psi_{0}= & e^{\left(t-t_{j-1}\right)\left(A+u_{j-1} B\right)} \circ \\
& \circ e^{\left(t_{j-1}-t_{j-2}\right)\left(A+u_{j-2} B\right)} \circ \cdots \circ e^{t_{0}\left(A+u_{0} B\right)} \psi_{0},
\end{aligned}
$$

for $t \in\left[t_{j-1}, t_{j}\right)$.

Remark 1: With extra regularity hypotheses, it is possible to define the propagator of (2) for a larger class of controls. For instance, when $B$ is bounded, for every $t, \Upsilon_{t}: u \mapsto \Upsilon_{t}^{u}$ admits a unique continuous extension to $L_{\mathrm{loc}}^{1}(\mathbf{R}, \mathbf{R})$.

\section{B. Control results}

Definition 1: Let $(A, B)$ satisfy Assumption 1. A subset $S$ of $\mathbf{N}^{2}$ couples two levels $j, k$ in $\mathbf{N}$, if there exists a finite sequence $\left(\left(s_{1}^{1}, s_{2}^{1}\right), \ldots,\left(s_{1}^{q}, s_{2}^{q}\right)\right)$ in $S$ such that

(i) $s_{1}^{1}=j$ and $s_{2}^{q}=k$;

(ii) $s_{2}^{j}=s_{1}^{j+1}$ for every $1 \leq j \leq q-1$;

(iii) $\left\langle\phi_{s_{1}^{j}}, B \phi_{s_{2}^{j}}\right\rangle \neq 0$ for $1 \leq j \leq q$.

The subset $S$ is called a connectedness chain for $(A, B)$ if $S$ couples every pair of levels in $\mathbf{N}$. A connectedness chain is said to be non-resonant if for every $\left(s_{1}, s_{2}\right)$ in $S, \mid \lambda_{s_{1}}-$ $\lambda_{s_{2}}|\neq| \lambda_{t_{1}}-\lambda_{t_{2}} \mid$ for every $\left(t_{1}, t_{2}\right)$ in $\mathbf{N}^{2} \backslash\left\{\left(s_{1}, s_{2}\right),\left(s_{2}, s_{1}\right)\right\}$ such that $\left\langle\phi_{t_{2}}, B \phi_{t_{1}}\right\rangle \neq 0$.

Definition 2: Let $(A, B)$ satisfy Assumption 1 The system $(A, B)$ is approximately simultaneously controllable if for every $\widehat{\Upsilon} \in U(H)$ (unitary operators acting on $H$ ), $\psi_{1}, \ldots, \psi_{n} \in H$, and $\varepsilon>0$, there exists a piecewise constant function $u_{\varepsilon}:\left[0, T_{\varepsilon}\right] \rightarrow \mathbf{R}$ such that

$$
\left\|\widehat{\Upsilon} \psi_{j}-\Upsilon_{T_{\varepsilon}}^{u_{\varepsilon}} \psi_{j}\right\|<\varepsilon .
$$

for every $j=1, \ldots, n$.

The following sufficient condition for approximate simultaneous controllability has been given in [9].

Proposition 1: Let $(A, B)$ satisfy Assumption 1 and admit a non-resonant chain of connectedness. Then $(A, B)$ is approximately simultaneously controllable.

\section{Weakly-coupled systems}

Definition 3: Let $k$ be a positive number and let $(A, B)$ satisfy Assumption 111. Then $(A, B)$ is $k$ weakly-coupled if for every $u \in \mathbf{R}, D\left(|A+u B|^{k / 2}\right)=D\left(|A|^{k / 2}\right)$ and there exists a constant $C$ such that, for every $\psi$ in $D\left(|A|^{k}\right)$, $\left|\Re\left\langle|A|^{k} \psi, B_{l} \psi\right\rangle\right| \leq C\left|\left\langle|A|^{k} \psi, \psi\right\rangle\right|$.

Definition 4: Let $N \in \mathbf{N}$. We define the projection $\pi_{N}$ : $\psi \in H \mapsto \sum_{k \leq N}\left\langle\phi_{k}, \psi\right\rangle \phi_{k}$. The Galerkin approximation of (2) of order $N$ is the system in $H$

$$
\dot{x}=\left(A^{(N)}+u B^{(N)}\right) x
$$

where $A^{(N)}=\pi_{N} A \pi_{N}$ and $B^{(N)}=\pi_{N} B \pi_{N}$ are the compressions of $A$ and $B$ (respectively).
We denote by $X_{(N)}^{u}(t, s)$ the propagator of $\left[\Sigma_{N}\right)$ associated with a piecewise constant functions $u$.

Proposition 2: Let $k$ and $s$ be non-negative numbers with $0 \leq s<k$. Let $(A, B)$ satisfy Assumption 1 and be $k$ weakly-coupled Assume that there exist $d>0,0 \leq r<k$ such that $\|B \psi\| \leq d\|\psi\|_{r / 2}$ for every $\psi$ in $D\left(|A|^{r / 2}\right)$. Then for every $\varepsilon>0, K \geq 0, n \in \mathbf{N}$, and $\left(\psi_{j}\right)_{1 \leq j \leq n}$ in $D\left(|A|^{k / 2}\right)^{n}$ there exists $N \in \mathbf{N}$ such that for every piecewise constant function $u$

$$
\|u\|_{L^{1}}<K \Longrightarrow\left\|\Upsilon_{t}^{u}\left(\psi_{j}\right)-X_{(N)}^{u}(t, 0) \pi_{N} \psi_{j}\right\|_{s / 2}<\varepsilon,
$$

for every $t \geq 0$ and $j=1, \ldots, n$.

\section{THE PERTURBED QUANTUM HARMONIC OSCILLATOR}

\section{A. Physical model}

The quantum harmonic oscillator is one of the most studied quantum system. Schrödinger equation reads

$$
i \frac{\partial \psi}{\partial t}(x, t)=-\frac{1}{2} \frac{\partial^{2} \psi}{\partial x^{2}}+\left(\frac{1}{2} x^{2}-u(t) x\right) \psi(x, t),
$$

where $x \in \Omega=\mathbf{R}$. With the notations of (2), $A=-i(-\Delta+$ $\left.x^{2}\right) / 2$ and $B=i x$.

An Hilbert basis of $H$ made of eigenvectors of $A$ is given by the sequence of the Hermite functions $\left(\phi_{n}\right)_{n \in \mathbf{N}}$, associated with the sequence $\left(-i \lambda_{n}\right)_{n \in \mathbf{N}}$ of eigenvalues where $\lambda_{n}=n-1 / 2$ for every $n$ in $\mathbf{N}$. In the basis $\left(\phi_{n}\right)_{n \in \mathbf{N}}$, $B$ admits a tri-diagonal structure:

$$
\left\langle\phi_{j}, B \phi_{k}\right\rangle=\left\{\begin{array}{cl}
-i \sqrt{k-1} & \text { if } j=k-1 \\
-i \sqrt{k} & \text { if } j=k+1 \\
0 & \text { otherwise, }
\end{array}\right.
$$

A chain of connectedness for this system is given by $S=$ $\{(n, n+1): n \in \mathbf{N}\}$. The chain $S$ is resonant indeed $\left|\lambda_{n+1}-\lambda_{n}\right|=1$ for every $n$ in $\mathbf{N}$. As a matter of fact, the system (3) is known to be non-controllable (see [14], [13]).

We consider a perturbation of this system. Consider the inverse $A^{-1}$ of the operator $A$. The family $\left(\phi_{n}\right)_{n \in \mathbf{N}}$ is a family of eigenvectors for $A^{-1}$ associated with the eigenvalues $\left(-i / \lambda_{n}\right)_{n \in \mathbf{N}}$. For every $\eta \geq 0$ we set $A_{\eta}=$ $A+\eta A^{-1}$. Since $A$ and $A^{-1}$ commute then $\left(\phi_{n}\right)_{n \in \mathbf{N}}$ is a family of eigenvectors for $A_{\eta}$ associated with the eigenvalues $\left(-i \lambda_{n}^{\eta}\right)_{n \in \mathbf{N}}$ where $\lambda_{n}^{\eta}=\lambda_{n}+\eta / \lambda_{n}$. The set $S$ is a nonresonant chain of connectedness for system $\left(A_{\eta}, B\right)$ for every $\eta>0$. Indeed $\lambda_{n+1}^{\eta}-\lambda_{n}^{\eta}=1-4 \frac{\eta}{4 n^{2}-1}$ and, clearly, $\lambda_{n+1}^{\eta}-\lambda_{n}^{\eta}=\lambda_{m+1}^{\eta}-\lambda_{m}^{\eta}$ if and only if $n=m$.

By Proposition 11 the system $\left(A_{\eta}, B\right)$ is approximately simultaneously controllable. Moreover by [9, Theorem 2.13] we have also an upper bound on the $L^{1}$-norm of the control independent of the error. For instance we can steer approximately the first level $\phi_{1}$ to the second $\phi_{2}$ with a control law with $L^{1}$-norm smaller than $5 \pi / 4$. Another consequence is that a quantum gate for $\phi_{1}, \phi_{2}$, and $\phi_{3}$ is approximately reachable, that is for every $\varepsilon>0$, there exists $t_{\varepsilon}>0$ and a piecewise constant function $u_{\varepsilon}$ such that $\| \Upsilon_{t_{\varepsilon}}^{u_{\varepsilon}}\left(\phi_{j}\right)-$ $\phi_{\sigma(j)} \|<\varepsilon$ where $\sigma$ is the 3 -cycle which exchanges 1,2 and 3 . This can be achieved with $\left\|u_{\varepsilon}\right\|_{L^{1}} \leq \pi / 2(1+\sqrt{2} / 2)$. 


\section{B. Estimates}

In the following, we only consider control of $L^{1}$-norm less than $K=\pi / 2(1+\sqrt{2} / 2)$. The particular tri-diagonal structure of system $\left(A_{\eta}, B\right)$ is very useful for a priori estimates on the components of the propagator. Indeed if $\|u\|_{L^{1}} \leq K$, by [10, Remark 6], we have that

$$
\left|\left\langle\phi_{n+1}, \Upsilon_{t}^{u}\left(\phi_{j}\right)\right\rangle\right| \leq \frac{(2 K)^{n-2}}{(n-2) !} \sqrt{\frac{(2 n-3) !}{(n-2) !}},
$$

for every $n$ in $\mathbf{N}, n \geq 3$ and $j=1,2,3$.

We use (4) to find estimates on the size $N$ of the Galerkin approximation whose existence is asserted by Proposition 2 .

First, let $N \geq j$ and notice that

$$
\begin{aligned}
\frac{d}{d t} \pi_{N} \Upsilon_{t}^{u}\left(\phi_{j}\right)=\left(A^{(N)}\right. & \left.+u B_{l}^{(N)}\right) \pi_{N} \Upsilon_{t}^{u}\left(\phi_{j}\right) \\
& +u(t) \pi_{N} B\left(\mathrm{Id}-\pi_{N}\right) \Upsilon_{t}^{u}\left(\phi_{j}\right) .
\end{aligned}
$$

Hence, by variation of constants, for every $t \geq 0$,

$$
\begin{aligned}
& \pi_{N} \Upsilon_{t}^{u}\left(\phi_{j}\right)=X_{(N)}^{u}(t, 0) \pi_{N} \phi_{j} \\
& \quad+\int_{0}^{t} X_{(N)}^{u}(t, s) \pi_{N} B\left(\operatorname{Id}-\pi_{N}\right) \Upsilon_{s}^{u}\left(\phi_{j}\right) u(\tau) d \tau
\end{aligned}
$$

Therefore, since $X_{(N)}^{u}(t, s)$ is unitary and for the tri-diagonal structure of the system we have, for $j=1,2,3$,

$$
\begin{aligned}
& \left\|\pi_{N} \Upsilon_{t}^{u}\left(\phi_{j}\right)-X_{(N)}^{u}(t, s) \phi_{j}\right\| \\
& \quad \leq K\left\|\pi_{N} B\left(\operatorname{Id}-\pi_{N}\right) \Upsilon_{t}^{u}\left(\phi_{j}\right)\right\| \\
& \quad=K\left|b_{N, N+1}\right|\left|\left\langle\phi_{N+1}, \Upsilon_{t}^{u}\left(\phi_{j}\right)\right\rangle\right| \\
& \quad=K \sqrt{N}\left|\left\langle\phi_{N+1}, \Upsilon_{t}^{u}\left(\phi_{j}\right)\right\rangle\right| \\
& \quad \leq \frac{2^{N-1} K^{N-1}}{(N-2) !} \sqrt{\frac{(2 N-3) !}{(N-3) !}}
\end{aligned}
$$

Using $K=\pi / 2(1+\sqrt{2} / 2)<2.69$, it is enough to consider a Galerkin approximation of size $N=420$ to get $\left\|\pi_{N} \Upsilon_{t}^{u}\left(\phi_{j}\right)-X_{(N)}^{u}(t, s) \phi_{j}\right\| \leq 10^{-4}$ for $j=1,2,3$.

\section{Numerical simulations}

For simulations, we choose $\eta=1$. To induce the transition between levels 1 and 2, the control law we use is a piecewise constant $4 \pi$ periodic function, taking value 1 for $0 \leq t<$ $5.10^{-3}$ and taking value 0 for $5.10^{-3} \leq t \leq 4 \pi$. We apply this control for 314 periods, that is during a time of $1256 \pi$.

To induce the transition between levels 2 and 3 , the control law we use is a piecewise constant $12 \pi / 5$ periodic function, taking value 1 for $0 \leq t<5 \cdot 10^{-3}$ and taking value 0 for $5.10^{-3} \leq t<12 \pi / 5$. We apply this control for 222 periods.

The simulations are done on a Galerkin approximation of size 420 , which garantees $\left\|\pi_{N} \Upsilon_{t}^{u}\left(\phi_{j}\right)-X_{(N)}^{u}(t, s) \phi_{j}\right\| \leq$ $10^{-4}$ for $j=1,2,3$. At final time $T$, the resulting propagator is such that $\left|\left\langle\Upsilon_{T}^{u} \phi_{1}, \phi_{3}\right\rangle\right|>0.998,\left|\left\langle\Upsilon_{T}^{u} \phi_{2}, \phi_{1}\right\rangle\right|>0.999$ and $\left|\left\langle\Upsilon_{T}^{u} \phi_{3}, \phi_{2}\right\rangle\right|>0.999$. The time evolution of the moduli of the first coordinates of $\Upsilon_{t}^{u}\left(\phi_{j}\right)$ for $j=1,2,3$ is depicted in Figures 1, 2, and 3.

All the computations were done using the free software NSP, see [12]. The source code for the simulation is available at [1].The total computation time is less than 4 minutes on a standard desktop computer.

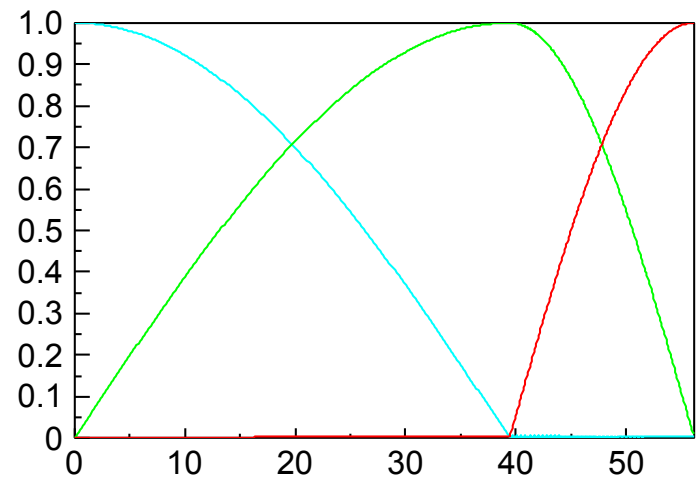

Fig. 1. Time evolution of the moduli of the first three coordinates of $\Upsilon_{t}^{u} \phi_{1}$ in the case of the perturbed harmonic oscillator. First coordinate in blue, second coordinate in green, third coordinate in red. For the sake of readability, time scale is $1: 100$, total duration around 5500 .

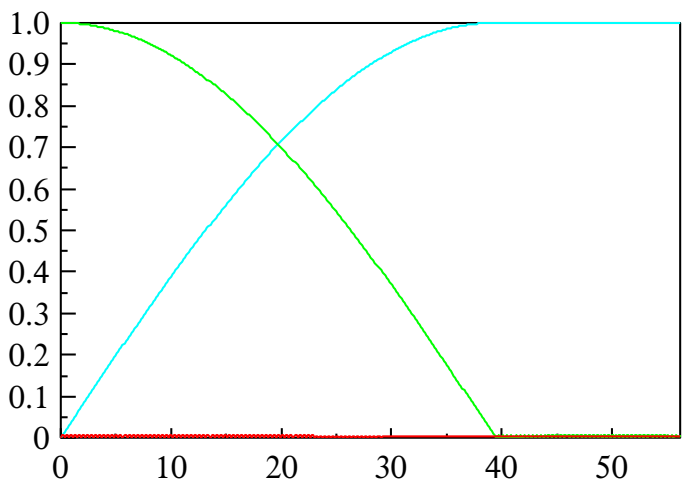

Fig. 2. Time evolution of the moduli of the first three coordinates of $\Upsilon_{t}^{u} \phi_{2}$ in the case of the perturbed harmonic oscillator. First coordinate in blue, second coordinate in green, third coordinate in red. Time scale is 1:100.

\section{PARTICLE IN A BOX}

\section{A. Physical model}

We consider now the case of a particle confined in $(0, \pi)$. This model has been extensively studied by several authors in the last few years and was the first quantum system for which a positive controllability result has been obtained. Beauchard proved exact controllability in some dense subsets of $L^{2}$ using Coron's return method (see [4], [6] for a precise statement). Nersesyan obtained approximate controllability results using Lyapunov techniques. In the following, we extend these controllability results to simultaneous controllability and provide some estimates of the $L^{1}$-norm of the controls achieving simultaneous controllability.

The Schrödinger equation writes

$$
i \frac{\partial \psi}{\partial t}=-\frac{1}{2} \frac{\partial^{2} \psi}{\partial x^{2}}-u(t) x \psi(x, t)
$$

with boundary conditions $\psi(0, t)=\psi(\pi, t)=0$ for every $t \in \mathbf{R}$. 


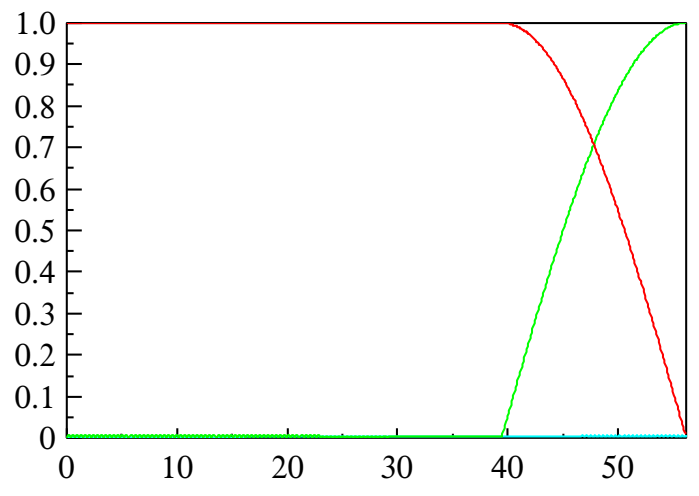

Fig. 3. Time evolution of the moduli of the first three coordinates of $\Upsilon_{t}^{u} \phi_{3}$ in the case of the perturbed harmonic oscillator. First coordinate in blue, second coordinate in green, third coordinate in red. Time scale is 1:100.

In this case $\mathcal{H}=L^{2}((0, \pi), \mathbb{C})$ endowed with the Hermitian product $\left\langle\psi_{1}, \psi_{2}\right\rangle=\int_{0}^{\pi} \overline{\psi_{1}(x)} \psi_{2}(x) d x$. The operators $A$ and $B$ are defined by $A \psi=i \frac{1}{2} \frac{\partial^{2} \psi}{\partial x^{2}}$ for every $\psi$ in $D(A)=\left(H_{2} \cap H_{0}^{1}\right)((0, \pi), \mathbb{C})$, and $B \psi=i x \psi$. An Hilbert basis of $H$ is $\left(\phi_{k}\right)_{k \in \mathbf{N}}$ with $\phi_{k}: x \mapsto \sin (k x) / \sqrt{2}$. For every $k, A \phi_{k}=-i k^{2} / 2 \phi_{k}$.

For every $j, k$ in $\mathbf{N}$,

$$
b_{j k}:=\left\langle\phi_{j}, B \phi_{k}\right\rangle= \begin{cases}(-1)^{j+k} \frac{2 j k}{\left(j^{2}-k^{2}\right)^{2}} & \text { if } j-k \text { odd } \\ 0 & \text { otherwise. }\end{cases}
$$

Despite numerous degenerate transitions, the system is approximately simultaneously controllable (see $[9$, Section 7]).

\section{B. Estimates}

Using Proposition 2 to estimate the error done when replacing infinite dimensional system by its Galerkin approximation one finds, for $\|u\|_{L^{1}}=9 \pi / 16$ (see [10, Remark 4], with $K=9 \pi / 16, d=\pi, k=1, r=1, c_{1}(A, B) \leq \pi+2$, $\varepsilon=10^{-3}$ ), that if $N>1.610^{7}$, then

$$
\left\|\pi_{N} \Upsilon_{t}^{u} \phi_{1}-X_{(N)}^{u}(t, 0) \phi_{1}\right\| \leq 10^{-3} .
$$

This estimation is definitely too rough to allow easy numerical simulations: matrix $B^{\left(10^{7}\right)}$ has about $510^{13}$ nonzeros entries, the numerical simulations at such scale are difficult without large computing facilities. We have to go more into details to obtain finer estimates.

Assume that, for some $N$ in $\mathbf{N}$ and $\eta>0$, the control $u:[0, T] \rightarrow \mathbf{R}$ is such that, for every $t$ in $[0, T]$,

$$
\left\|X_{(N)}^{u}(t, 0) \pi_{3}-\pi_{3} X_{(N)}^{u}(t, 0)\right\| \leq \eta .
$$

We have

$$
\begin{aligned}
& \pi_{3} X_{(N)}^{u}(t, s)-X_{(N)}^{u}(t, s) \pi_{3} \\
& =\quad \pi_{3} X_{(N)}^{u}(t, 0) X_{(N)}^{u}(0, s)-X_{(N)}^{u}(t, 0) X_{(N)}^{u}(0, s) \pi_{3} \\
& =\quad X_{(N)}^{u}(t, 0)\left(\pi_{3} X_{(N)}^{u}(0, s)-X_{(N)}^{u}(0, s) \pi_{3}\right) \\
& \quad \quad+\left(\pi_{3} X_{(N)}^{u}(t, 0)-X_{(N)}^{u}(t, 0) \pi_{3}\right) X_{(N)}^{u}(0, s)
\end{aligned}
$$

so that

$$
\left\|\pi_{3} X_{(N)}^{u}(t, s)-X_{(N)}^{u}(t, s) \pi_{3}\right\| \leq 2 \eta .
$$

Projecting (5) on the first 3 components we have, for $j=$ $1,2,3$ that

$$
\begin{aligned}
\left\|\pi_{3} \Upsilon_{t}^{u}\left(\phi_{j}\right)-\pi_{3} X_{(N)}^{u}(t, 0) \phi_{j}\right\| & \\
\leq & \int_{0}^{t}\left\|\pi_{3} X_{(N)}^{u}(t, s) \pi_{N} B\left(\mathrm{Id}-\pi_{N}\right) \Upsilon_{s}^{u}\left(\phi_{j}\right)\right\| u(s) \mathrm{d} s \\
\leq & \int_{0}^{t}\left\|X_{(N)}^{u}(t, s) \pi_{3} B\left(\mathrm{Id}-\pi_{N}\right) \Upsilon_{s}^{u}\left(\phi_{j}\right)\right\| u(s) \mathrm{d} s \\
& \quad+\int_{0}^{t}\left\|\left(\pi_{3} X_{(N)}^{u}(t, s)-X_{(N)}^{u}(t, s) \pi_{3}\right)\right\|\|B\| u(s) \mathrm{d} s \\
\leq & \quad\left(\int_{0}^{T}|u(t)| \mathrm{d} t\right)\left(\left\|\pi_{3} B\left(\mathrm{Id}-\pi_{N}\right)\right\|\right. \\
& \left.\left.\quad+2\|B\| \sup _{t} \| \pi_{3} X_{(N)}^{u}(t, 0)-X_{(N)}^{u}(t, 0) \pi_{3}\right) \|\right) .(8)
\end{aligned}
$$

By skew-adjointness, $\left\|\pi_{3} B\left(\mathrm{Id}-\pi_{N}\right)\right\|=\left\|\left(\mathrm{Id}-\pi_{N}\right) B \pi_{3}\right\|$. This last quantity tends to zero, and we are able to give estimates of the convergence rate. Indeed,

$$
\begin{aligned}
\left\|\left(\mathrm{Id}-\pi_{N}\right) B \phi_{1}\right\|^{2} & \leq \sum_{k>N}\left|\frac{2 k}{(k-1)^{2}(1+k)^{2}}\right|^{2} \\
& \leq 4 \sum_{k>N} \frac{1}{(k-1)^{6}} \\
& \leq \frac{1}{(N-2)^{5}} .
\end{aligned}
$$

Similarly,

$$
\begin{aligned}
\left\|\left(\mathrm{Id}-\pi_{N}\right) B \phi_{2}\right\|^{2} & \leq \frac{\sqrt{2}}{(N-3)^{5}} \\
\left\|\left(\mathrm{Id}-\pi_{N}\right) B \phi_{3}\right\|^{2} & \leq \frac{2}{(N-4)^{5}} .
\end{aligned}
$$

The procedure to induce a given transformation, up to a given tolerance $\varepsilon>0$, on the space $\operatorname{span}\left\{\phi_{1}, \phi_{2}, \phi_{3}\right\}$ is the following:

1) Use estimates given in [9] to give an a priori upper bound $K$ on the $L^{1}$-norm of the controls one will use.

2) From $K$ and $\varepsilon$, find $N$ such that $K\left\|\pi_{3} B\left(\operatorname{Id}-\pi_{N}\right)\right\| \leq$ $\varepsilon / 2$.

3) In the finite dimensional space $\operatorname{span}\left\{\phi_{1}, \ldots, \phi_{N}\right\}$, consider the bilinear system $\dot{x}=\left(A^{(N)}+u B^{(N)}\right) x$ and find a control $u$ achieving the desired transition up to $\varepsilon /(2 K)$ and such that $\|u\|_{L^{1}} \leq K$. This can be done using standard averaging procedures (see for instance [15]).

4) Use (8) to get an upper bound of the distance of the trajectories of $\left(\Sigma_{N}\right)$ and the actual infinite dimensional system.

\section{Numerical simulations}

We illustrate the above procedure on an example. Fix $\varepsilon=710^{-2}$. We would like to find $u:[0, T] \rightarrow \mathbf{R}$ such that $\left|\left\langle\phi_{3}, \Upsilon_{T}^{u} \phi_{1}\right\rangle\right|>1-\varepsilon,\left|\left\langle\phi_{1}, \Upsilon_{T}^{u} \phi_{2}\right\rangle\right|>1-\varepsilon$ and 


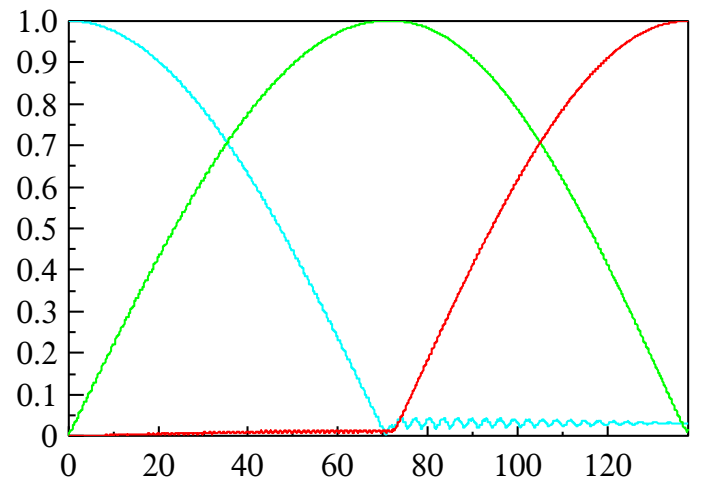

Fig. 4. Time evolution of the moduli of the first three coordinates of $\Upsilon_{t}^{u} \phi_{1}$ in the case of the potential well. First coordinate in blue, second coordinate in green, third coordinate in red.

$\left|\left\langle\phi_{2}, \Upsilon_{T}^{u} \phi_{3}\right\rangle\right|>1-\varepsilon$ at final time $T$. For this example, we are not interested in the respective phases but the method can easily be generalized to address this point (see Section IV-D below).

From [9], the transition can be achieved with controls of $L^{1}$-norm smaller than $5 \pi / 4(9 / 8+25 / 24)$. Using controls with better efficiencies (as described in [11]), we can use controls with $L^{1}$-norm smaller than $2(9 / 8+25 / 4)=13 / 3$.

Using the above estimates, one sees that if $N=20$, then

$$
K\left\|\pi_{3} B\left(\mathrm{Id}-\pi_{N}\right)\right\| \leq \frac{13}{3} \frac{\sqrt{2}}{(N-4)^{5 / 2}} \leq 610^{-3} .
$$

Last, we define $u$ by $u(t)=\cos (3 t) / 20$ for $0 \leq t \leq$ 72 and $u(t)=\cos (5 t) / 20$ for $72<t \leq T=138$. We check that $\int_{0}^{T}|u(t)| \mathrm{d} t \leq 13 / 3$. One checks numerically that $\left\|\pi_{3} X_{(20)}^{u}(t, 0)-X_{(20)}^{u}(t, 0) \pi_{3}\right\| \leq 1.310^{-3}$ for $t \leq 138$. From [7, we get, for every $t, s \leq T$

$$
\left\|\pi_{3} X_{(N)}^{u}(t, s)-X_{(N)}^{u}(t, s) \pi_{3}\right\| \leq 2.610^{-3} \text {. }
$$

From [8], we have, for $j=1,2,3$,

$$
\begin{aligned}
\left\|\pi_{3} \Upsilon_{t}^{u}\left(\phi_{j}\right)-\pi_{3} X_{(N)}^{u}(t, 0) \phi_{j}\right\| & \leq \frac{13}{3}\left(610^{-3}+8.210^{-3}\right) \\
& \leq 6.110^{-2} .
\end{aligned}
$$

Conclusion follows from the numerical computations

$$
\begin{aligned}
\left|\left\langle\phi_{3}, X_{(20)}^{u}(T, 0) \phi_{1}\right\rangle\right| & \approx 0.99924 \\
\left|\left\langle\phi_{1}, X_{(20)}^{u}(T, 0) \phi_{2}\right\rangle\right| & \approx 0.99943 \\
\left|\left\langle\phi_{2}, X_{(20)}^{u}(T, 0) \phi_{3}\right\rangle\right| & \approx 0.99949 .
\end{aligned}
$$

The actual precision is likely much better than $6.110^{-2}$ which is known for sure. However, our estimates do not allow a better conclusion.

The evolutions with respect to the time of the moduli of the first coordinates of $X_{(20)}^{u} \phi_{k}$ for $k=1,2,3$ are represented in Figures 4, 5, and 6.

All the computations were done using the free software NSP, see [12]. The source code for the simulation is available at [2]. The total computation time is less than 4 minutes on a standard desktop computer.

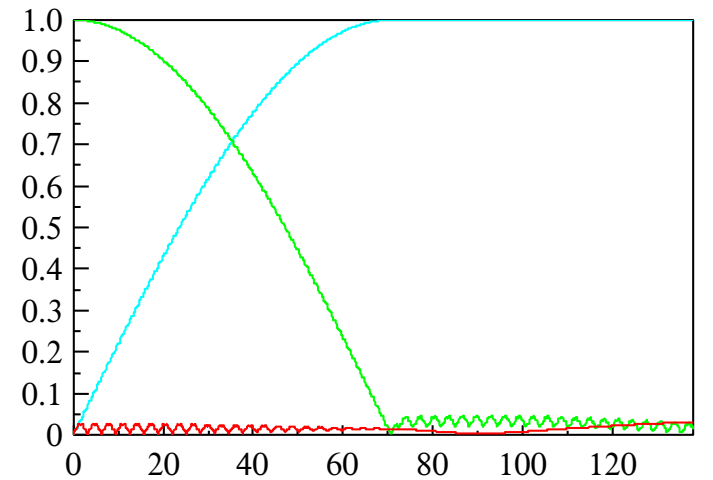

Fig. 5. Time evolution of the moduli of the first three coordinates of $\Upsilon_{t}^{u} \phi_{2}$ in the case of the potential well. First coordinate in blue, second coordinate in green, third coordinate in red.

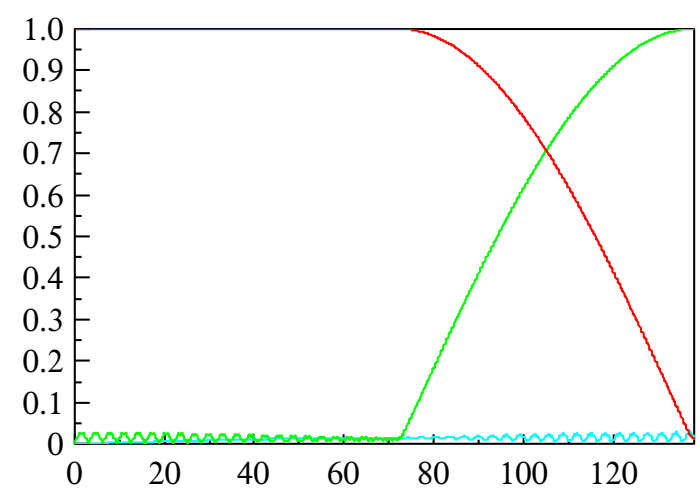

Fig. 6. Time evolution of the moduli of the first three coordinates of $\Upsilon_{t}^{u} \phi_{3}$ in the case of the potential well. First coordinate in blue, second coordinate in green, third coordinate in red.

\section{Possible improvements}

If one is interested not only in the modulus but also in the respective phases of the final points, it is enough to replace the functions $t \mapsto \cos (3 t) / 20$ and $t \mapsto \cos (5 t) / 20$ above by $t \mapsto \cos \left(3 t+\theta_{1}\right) / 20$ and $t \mapsto \cos \left(5 t+\theta_{2}\right) / 20$ respectively, where $\theta_{1}$ and $\theta_{2}$ are suitable phases.

In order to get better precision in the approximation (i.e. a smaller $\varepsilon$ ), it is enough to replace the functions $t \mapsto$ $\cos (3 t) / 20$ and $t \mapsto \cos (5 t) / 20$ above by the functions $t \mapsto \cos (3 t) / L$ and $t \mapsto \cos (5 t) / L$ with $L$ large enough. The price to pay for a better precision is an increase in the time needed for the transfer.

\section{CONCLUSION AND FUTURE WORKS}

We have shown how it was possible to implement a quantum gate on two types of infinite dimensional quantum oscillators. Our method provides rigorous estimates and permits numerical simulations that can be run on standard desktop computers.

A limitation of our models is that the Schrödinger equation neglects decoherence. This approximation may be justified 
for time small with respect to the relaxation time of the quantum system. Future works may focus on the optimization of the time of implementation.

\section{REFERENCES}

[1] Source code for the simulations of the present paper (harmonic oscillator). http://www.iecn.u-nancy.fr/ chambrio/ Fichiers/Harmonicoscillator.sce

[2] Source code for the simulations of the present paper (potential well). http://www.iecn.u-nancy.fr/ chambrio/ Fichiers/PotentialWell.sce

[3] J. M. Ball, J. E. Marsden, and M. Slemrod. Controllability for distributed bilinear systems. SIAM J. Control Optim., 20(4):575-597, 1982.

[4] Karine Beauchard and Jean-Michel Coron. Controllability of a quantum particle in a moving potential well. J. Funct. Anal., 232(2):328389, 2006.

[5] Karine Beauchard and Camille Laurent. Local controllability of 1D linear and nonlinear Schrödinger equations with bilinear control. J. Math. Pures Appl., 94(5):520-554, 2010.

[6] Karine Beauchard and Mazyar Mirrahimi. Practical stabilization of a quantum particle in a one-dimensional infinite square potential well. SIAM J. Control Optim., 48(2):1179-1205, 2009.
[7] Karine Beauchard and Vahagn Nersesyan. Semi-global weak stabilization of bilinear Schrödinger equations. arXiv:1005.4558v1.

[8] Mohamed Belhadj, Julien Salomon, and Gabriel Turinici. A stable toolkit method in quantum control. J. Phys. A, 41(36):362001, 10, 2008.

[9] Ugo Boscain, Marco Caponigro, Thomas Chambrion, and Mario Sigalotti. A weak spectral condition for the controllability of the bilinear Schrödinger equation with application to the control of a rotating planar molecule. arXiv:1101.4313v1, 2011.

[10] Nabile Boussaid, Marco Caponigro, and Thomas Chambrion. Weaklycoupled systems in quantum control. arXiv: 1109.1900.

[11] Thomas Chambrion. Periodic excitations of bilinear quantum systems. arXiv: 1103.1130

[12] A free software for numerical computations. Nsp. http:// cermics.enpc.fr/ jpc/nsp-tiddly/

[13] Reinhard Illner, Horst Lange, and Holger Teismann. Limitations on the control of Schrödinger equations. ESAIM Control Optim. Calc. Var., 12(4):615-635 (electronic), 2006

[14] Mazyar Mirrahimi and Pierre Rouchon. Controllability of quantum harmonic oscillators. IEEE Trans. Automat. Control, 49(5):745-747, 2004.

[15] Jan Sanders and Ferdinand Verhulst. Averaging methods in nonlinear dynamical systems, volume 59 of Applied Mathematical Sciences. Springer, New York, second edition, 2007. 